\title{
A Meshfree Method for Signorini Problems Using Boundary Integral Equations
}

\author{
Yanlin Ren and Xiaolin Li \\ College of Mathematics Science, Chongqing Normal University, Chongqing 400047, China \\ Correspondence should be addressed to Xiaolin Li; lxlmath@163.com
}

Received 5 November 2013; Accepted 1 January 2014; Published 18 February 2014

Academic Editor: Khai Ching Ng

Copyright ( 2014 Y. Ren and X. Li. This is an open access article distributed under the Creative Commons Attribution License, which permits unrestricted use, distribution, and reproduction in any medium, provided the original work is properly cited.

\begin{abstract}
This paper presents a meshfree method for the numerical solution of Signorini problems. In this method, a projection iterative algorithm is used to convert the boundary inequality constraints into a fixed point equation. Then, the boundary value problem is reformulated as boundary integral equations and the unknown boundary variables are interpolated by the point interpolation scheme. Thus, only a nodal data structure on the boundary of a domain is required, and boundary conditions can be implemented directly and easily. The convergence of this method is verified theoretically. Numerical examples involving groundwater flow and electropainting problems are also provided to illustrate the performance and usefulness of the method.
\end{abstract}

\section{Introduction}

Signorini problems arise in the modeling of many real world engineering applications such as the shallow dam problem [1-3], the electropaint process $[1,3,4]$, the fluid mechanics problems in media with semipermeable boundaries [5], and free surface problems [6]. These problems form a special class of boundary value problems, since the boundary potential and its normal derivative alternate in conjunction with certain boundary inequality constraints. The main difficulty in tackling Signorini problems comes from the fact that the position where the change from one type of condition to the other occurs is unknown in advance. The numerical solution of Signorini problems has been frequently dominated by classical numerical methods such as the finite difference method, the finite element method (FEM) $[2,4,5]$, and the boundary element method (BEM) [1,7-9]. Although these methods have been successfully applied to many engineering situations, their accuracy depends critically on the generation of meshes, which can be time consuming, arduous, and fraught with pitfalls.

In the past two decades, there has been growing interest in developing meshfree (or meshless) methods, for the simple reason that, as compared to mesh-based methods such as the FEM and the BEM, one requires only nodes rather than elements $[10,11]$. Some meshfree methods, such as the element free Galerkin (EFG) method, the reproducing kernel particle method, the meshless local Petrov-Galerkin method, the point interpolation method, the radial basis collocation method [12], the smoothed particle hydrodynamics (SPH) method [13], and the moving particle semi-implicit (MPS) method [14], have been developed for real world physical problems. They are of domain type, as the FEM, in which the problem domain is discretized by nodes.

Boundary integral equations (BIEs) are attractive computational techniques as they can reduce the dimensionality of the original problem by one. The idea of meshfree has also been applied in boundary integral equations (BIEs) $[10,11,15-23]$. The first BIEs-based meshfree method is the boundary node method (BNM) $[10,15]$, which takes the advantages of both BIEs in dimension reduction and moving least-squares (MLS) approximations in elements elimination. However, since the MLS approximations lack the delta function property, boundary conditions in the BNM cannot be enforced easily.

The point interpolation method (PIM) $[11,24]$ is another meshfree interpolation technique that uses polynomial basis to construct meshfree shape functions. The PIM approximates its field variable by letting the problem function pass through each scattered node in its local support 
domain and polynomial basis is used for point interpolation. Different from the MLS approximation, the shape functions so constructed have delta function properties. Some meshfree methods using the PIM shape functions have been developed. Among them are the PIM [24], the radial PIM [25], the boundary PIM [21, 22], the hybrid boundary PIM [23], and the hybrid radial BNM [20]. In these PIM-based methods, boundary conditions can be enforced as easily as in the conventional FEM and BEM.

This paper is a first attempt in implementing BIEs-based meshfree method to Signorini problems. With the help of a fixed point equation and a projection iterative algorithm [26-28], the original Signorini problem is converted into a projection iterative problem. Then, regularized BIE is used to express the solution of the iterative problem, while the PIM is used to interpolate the unknown boundary variables. In this method, boundary inequality constraints are incorporated into the iterative scheme naturally, and boundary conditions can be applied with ease. Besides, since the Signorini boundary is of prime interest, this method is very suitable for Signorini problems. The convergence proof of this meshfree method is also derived mathematically. The accuracy and efficiency of this method are demonstrated by means of validation results for an annular problem with analytical solutions and test problems for the shallow dam problem and the electropainting problem.

The rest of this paper is organized as follows. Section 2 describes the boundary point interpolation scheme. In Section 3, detailed numerical implementation of our meshfree method is described for solving Signorini problems. Section 4 presents the convergence analysis of this method. Numerical examples are given in Section 5. Finally, the conclusion is presented in Section 6.

\section{Boundary Variables Interpolation}

Let $\Omega$ be a bounded domain in $\mathbb{R}^{2}$ with smooth boundary $\Gamma$. A generic point in $\mathbb{R}^{2}$ is denoted by $\mathbf{x}=\left(x_{1}, x_{2}\right)$ or $\mathbf{y}=\left(y_{1}, y_{2}\right)$. Let $\mathbf{n}=\left(n_{1}, n_{2}\right)$ denote the outward normal direction on $\Gamma$.

A point interpolation scheme on a generic bounding surface $\Gamma$ is presented here. Since the nodes lie on $\Gamma$ in the present method, the point interpolation is only needed on $\Gamma$. As in $[10,18-20]$, it is assumed that the boundary $\Gamma$ is the union of piecewise smooth segments $\Gamma_{i}\left(i=1,2, \ldots, N_{\Gamma}\right)$ which constitute the boundary naturally. The segment can be obtained easily, since the only restriction is that each segment is smooth and the union of all the segments equals the boundary $\Gamma$. For example, each side of a polygon is a segment. The difference in the point interpolation between the present method and other PIM-based methods [21-23] is that, in the present method, the point interpolation is constructed on each segment independently other than the whole boundary $\Gamma$. Thus, the discontinuity at corners is avoided.

In the following interpolation scheme, let the variable $v$ denote the potential $u$ or its normal derivative $q=\partial u / \partial \mathbf{n}$ for simplicity. In an interpolation domain with $N_{I}$ scattered nodes, the function $v$ can be approximated as

$$
v(s)=\sum_{i=1}^{N_{I}} a_{i} p_{i}(s)=\mathbf{p}^{\mathrm{T}}(s) \mathbf{a},
$$

where $s$ is a parametric coordinate on $\Gamma, p_{1}=1$ and $p_{j}(s)(j=$ $\left.2,3, \ldots, N_{I}\right)$ are monomials in $s$, and $a_{i}$ is the interpolation coefficient,

$$
\begin{gathered}
\mathbf{p}^{\mathrm{T}}(s)=\left[p_{1}(s), p_{2}(s), \ldots, p_{N_{I}}(s)\right], \\
\mathbf{a}=\left[a_{1}, a_{2}, \ldots, a_{N_{I}}\right]^{T} .
\end{gathered}
$$

The unknown coefficient $a_{i}$ is normally solved via collocation,

$$
v\left(s_{k}\right)=\mathbf{p}^{T}\left(s_{k}\right) \mathbf{a}, \quad 1 \leq k \leq N_{I} .
$$

It is represented in matrix form as follows:

$$
\mathbf{v}=\mathbf{P a},
$$

where the vector of nodal function values is given by

$$
\mathbf{v}=\left[v\left(s_{1}\right), v\left(s_{2}\right), \ldots, v\left(s_{N_{I}}\right)\right]^{\mathrm{T}}
$$

and the coefficient matrix is

$$
\mathbf{P}=\left[\mathbf{p}\left(s_{1}\right), \mathbf{p}\left(s_{2}\right), \ldots, \mathbf{p}\left(s_{N_{I}}\right)\right]^{\mathrm{T}} .
$$

Solving (4) yields

$$
\mathbf{a}=\mathbf{P}^{-1} \mathbf{v}
$$

Then substituting (7) into (1), we obtain

$$
v(s)=\mathbf{p}^{\mathrm{T}}(s) \mathbf{P}^{-1} \mathbf{v}=\boldsymbol{\Phi}(s) \mathbf{v},
$$

where the shape function vector is

$$
\boldsymbol{\Phi}(s)=\mathbf{p}^{\mathrm{T}}(s) \mathbf{P}^{-1}=\left[\varphi_{1}(s), \varphi_{2}(s), \ldots, \varphi_{i}(s), \ldots, \varphi_{N_{I}}(s)\right] .
$$

Obviously, the meshfree shape function obtained through the above process possesses a delta function property.

\section{Meshfree Formulations for Signorini Problems}

3.1. Signorini Problems. Consider the following Signorini problem:

$$
\begin{gathered}
\Delta u=0, \quad \text { in } \Omega, \\
u=\bar{u}, \quad \text { on } \Gamma_{D}, \\
q:=\frac{\partial u}{\partial \mathbf{n}}=\bar{q}, \quad \text { on } \Gamma_{N},
\end{gathered}
$$


and the following Signorini boundary conditions:

$$
u \geq h, \quad q \geq g, \quad(u-h)(q-g)=0, \quad \text { on } \Gamma_{S},
$$

where the boundary $\Gamma$ consists of three parts, Dirichlet boundary $\Gamma_{D}$, Neumann boundary $\Gamma_{N}$, and Signorini boundary $\Gamma_{S}, u$ is an unknown function to be determined, $\mathbf{n}$ is the unit outward normal to $\Gamma, q$ is the normal derivative of $u, h$ and $g$ are prescribed functions on $\Gamma_{S}$, and $\bar{u}$ and $\bar{q}$ are the prescribed potential and normal flux on $\Gamma_{D}$ and on $\Gamma_{N}$, respectively. It is assumed that $\Gamma_{S}$ is not empty. In Signorini problems, we need to determine on which part of $\Gamma_{S}$ boundary conditions

$$
u=h, \quad q \geq g
$$

are satisfied, and then, on the remaining parts of $\Gamma_{S}$, boundary conditions

$$
u \geq h, \quad q=g
$$

are satisfied.

3.2. Projection Iterative Scheme. The problem of satisfaction of Signorini boundary conditions can be transferred to a fixed point problem [26-28]. In order to do so, we define the projection operator

$$
[v]_{+}=\max (0, v), \quad v \in \mathbb{R},
$$

and consider the following fixed point equation:

$$
(q-g)-[(q-g)-c(u-h)]_{+}=0, \quad \text { on } \Gamma_{S},
$$

where $c$ is any positive constant. Since (17) implies that $\min \{u-h, q-g\}=0$, the fixed point equation is equivalent to the Signorini boundary conditions. This alternative formulation is very important from the numerical analysis point of view. Consequently, we have the following theorem.

Theorem 1. The original Signorini problem given by (10)-(13) is equivalent to the following boundary value problem:

$$
\begin{gathered}
\Delta u=0, \quad \text { in } \Omega, \\
u=\bar{u}, \quad \text { on } \Gamma_{D}, \\
q=\bar{q}, \quad \text { on } \Gamma_{N}, \\
(q-g)-[(q-g)-c(u-h)]_{+}=0, \quad \text { on } \Gamma_{S} .
\end{gathered}
$$

To tackle the fixed point equation (17) numerically, we define the following remnant function:

$$
R(u, q)=(q-g)-[(q-g)-c(u-h)]_{+}, \quad \text { on } \Gamma_{S} .
$$

Obviously, $(u, q)$ is a zero of $R(u, q)=0$ if and only if $(u, q)$ is the solution of the following equation:

$$
\begin{aligned}
(q-g)+c(u-h)= & (q-g)+c(u-h) \\
& -\omega R(u, q), \text { on } \Gamma_{S},
\end{aligned}
$$

where $\omega \in(0,2)$. Using $(20)$, we define an iterative form

$$
\begin{aligned}
\left(q^{(k+1)}-g\right)+c\left(u^{(k+1)}-h\right)= & \left(q^{(k)}-g\right)+c\left(u^{(k)}-h\right) \\
& -\omega R\left(u^{(k)}, q^{(k)}\right), \text { on } \Gamma_{S} .
\end{aligned}
$$

Namely,

$$
q^{(k+1)}+c u^{(k+1)}=q^{(k)}+c u^{(k)}-\omega R\left(u^{(k)}, q^{(k)}\right), \quad \text { on } \Gamma_{S},
$$

where $k=0,1,2, \ldots$. Then, inserting (22) into (18) yields the following projection iterative problem:

$$
\begin{gathered}
\Delta u^{(k+1)}=0, \quad \text { in } \Omega, \\
u^{(k+1)}=\bar{u}, \quad \text { on } \Gamma_{D}, \\
q^{(k+1)}=\bar{q}, \quad \text { on } \Gamma_{N}, \\
q^{(k+1)}+c u^{(k+1)}=q^{(k)}+c u^{(k)}-\omega R\left(u^{(k)}, q^{(k)}\right), \quad \text { on } \Gamma_{S} .
\end{gathered}
$$

From (23), we can obtain the unknown potential $u^{(k+1)}$ and its normal derivative $q^{(k+1)}$ on $\Gamma_{S}$.

3.3. Discretization. The boundary integral expression for the solution of (23) can be expressed as

$$
\begin{array}{r}
\int_{\Gamma} u^{*}(\mathbf{x}, \mathbf{y}) q^{(k+1)}(\mathbf{y}) \mathrm{d} \Gamma \\
-\int_{\Gamma} q^{*}(\mathbf{x}, \mathbf{y})\left[u^{(k+1)}(\mathbf{y})-u^{(k+1)}(\mathbf{x})\right] \mathrm{d} \Gamma=0, \\
\mathbf{x} \in \Gamma,
\end{array}
$$

where $u^{*}(\mathbf{x}, \mathbf{y})=-(1 / 2 \pi) \ln |\mathbf{x}-\mathbf{y}|$ is the fundamental solution of the Laplace operator and has weak singularity on the diagonal $\{(\mathbf{x}, \mathbf{y}) \in \Gamma \times \Gamma: \mathbf{x}=\mathbf{y}\}$ and $q^{*}(\mathbf{x}, \mathbf{y})=$ $\partial u^{*}(\mathbf{x}, \mathbf{y}) / \partial \mathbf{n}_{\mathbf{y}}$ is the strongly singular fundamental solution.

Let $\left\{\mathbf{x}_{i}\right\}_{i=1}^{N}$ be a set of $N$ boundary nodes $\mathbf{x}_{i} \in \Gamma$. According to (8), the interpolation of $u^{(k+1)}$ and $q^{(k+1)}$ can be written as

$$
\begin{aligned}
& u^{(k+1)}(\mathbf{x})=\sum_{j=1}^{N} \phi_{j}(\mathbf{x}) u_{j}^{(k+1)}, \\
& q^{(k+1)}(\mathbf{x})=\sum_{j=1}^{N} \phi_{j}(\mathbf{x}) q_{j}^{(k+1)},
\end{aligned}
$$

where $u_{j}^{(k+1)}$ and $q_{j}^{(k+1)}$ are the nodal values $u^{(k+1)}\left(\mathbf{x}_{j}\right)$ and $q^{(k+1)}\left(\mathbf{x}_{j}\right)$, respectively, at the boundary node $\mathbf{x}_{j}$ and $\phi_{j}(\mathbf{x})$ is the contributions from the node $\mathbf{x}_{j}$ to the evaluation point $\mathbf{x}$. In the influence domain of the $j$ th node, $\phi_{j}(\mathbf{x})$ is the meshfree shape function given by (9) and is not equal to zero. Otherwise, $\phi_{j}(\mathbf{x})$ is equal to zero. 
Inserting (25) into (24) for all boundary nodes $\left\{\mathbf{x}_{i}\right\}_{i=1}^{N}$ yields

$$
\begin{aligned}
& \sum_{j=1}^{N} q_{j}^{(k+1)} \int_{\Gamma} u^{*}\left(\mathbf{x}_{i}, \mathbf{y}\right) \phi_{j}(\mathbf{y}) \mathrm{d} \Gamma \\
& \quad-\sum_{j=1}^{N} u_{j}^{(k+1)} \int_{\Gamma} q^{*}\left(\mathbf{x}_{i}, \mathbf{y}\right)\left[\phi_{j}(\mathbf{y})-\phi_{j}\left(\mathbf{x}_{i}\right)\right] \mathrm{d} \Gamma \\
& \quad=0 .
\end{aligned}
$$

It is represented in matrix form as follows:

$$
\mathrm{HU}=\mathrm{GQ} \text {, }
$$

where $\mathbf{H}$ and $\mathbf{G}$ are the corresponding coefficient matrices and

$$
\begin{gathered}
\mathbf{U}=\left(u_{1}^{(k+1)}, u_{2}^{(k+1)}, \ldots, u_{N}^{(k+1)}\right)^{\mathrm{T}}, \\
\mathbf{Q}=\left(q_{1}^{(k+1)}, q_{2}^{(k+1)}, \ldots, q_{N}^{(k+1)}\right)^{\mathrm{T}}, \\
H_{i j}=\int_{\Gamma} q^{*}\left(\mathbf{x}_{i}, \mathbf{y}\right)\left[\phi_{j}(\mathbf{y})-\phi_{j}\left(\mathbf{x}_{i}\right)\right] \mathrm{d} \Gamma, \\
G_{i j}=\int_{\Gamma} u^{*}\left(\mathbf{x}_{i}, \mathbf{y}\right) \phi_{j}(\mathbf{y}) \mathrm{d} \Gamma .
\end{gathered}
$$

To carry out integrations numerically, the boundary $\Gamma$ is discretized into cells. It has been shown that one node per cell and the position of the node at the center of the cell can yield excellent results $[15,18,19]$. This nodal placement is used in this research.

3.4. Imposition of Boundary Conditions and Assembly of Equations. To simplify the representation, we further assume that the first $N_{d}$ boundary nodes $\left\{\mathbf{x}_{i}\right\}_{i=1}^{N_{d}}$ belong to $\Gamma_{D}$, the next $N_{n}$ boundary nodes $\left\{\mathbf{x}_{i}\right\}_{i=N_{d}+1}^{N_{d}+N_{n}}$ belong to $\Gamma_{N}$, and the rest of $N_{s}=N-N_{d}-N_{n}$ boundary nodes $\left\{\mathbf{x}_{i}\right\}_{i=N_{d}+N_{n}+1}^{N}$ belong to $\Gamma_{S}$. Then, by applying the Dirichlet and Neumann boundary condition given in (23), we can rewrite (27) as the following block form:

$$
\left[\begin{array}{lll}
\mathbf{H}_{11} & \mathbf{H}_{12} & \mathbf{H}_{13} \\
\mathbf{H}_{21} & \mathbf{H}_{22} & \mathbf{H}_{23} \\
\mathbf{H}_{31} & \mathbf{H}_{32} & \mathbf{H}_{33}
\end{array}\right]\left[\begin{array}{l}
\overline{\mathbf{U}}_{1} \\
\mathbf{U}_{2} \\
\mathbf{U}_{3}
\end{array}\right]=\left[\begin{array}{lll}
\mathbf{G}_{11} & \mathbf{G}_{12} & \mathbf{G}_{13} \\
\mathbf{G}_{21} & \mathbf{G}_{22} & \mathbf{G}_{23} \\
\mathbf{G}_{31} & \mathbf{G}_{32} & \mathbf{G}_{33}
\end{array}\right]\left[\begin{array}{l}
\mathbf{Q}_{1} \\
\overline{\mathbf{Q}}_{2} \\
\mathbf{Q}_{3}
\end{array}\right],
$$

where the vectors $\overline{\mathbf{U}}_{1}=\left(u_{1}^{(k+1)}, u_{2}^{(k+1)}, \ldots, u_{N_{d}}^{(k+1)}\right)^{\mathrm{T}}$ and $\overline{\mathbf{Q}}_{2}=\left(q_{N_{d}+1}^{(k+1)}, q_{N_{d}+2}^{(k+1)}, \ldots, q_{N_{d}+N_{n}}^{(k+1)}\right)^{\mathrm{T}}$ are given, and the vectors $\mathbf{Q}_{1}=\left(q_{1}^{(k+1)}, \ldots, q_{N_{d}}^{(k+1)}\right)^{\mathrm{T}}, \mathbf{U}_{2}=\left(u_{N_{d}+1}^{(k+1)}, \ldots, u_{N_{d}+N_{n}}^{(k+1)}\right)^{\mathrm{T}}, \mathbf{U}_{3}=$ $\left(u_{N_{d}+N_{n}+1}^{(k+1)}, \ldots, u_{N}^{(k+1)}\right)^{\mathrm{T}}$, and $\mathbf{Q}_{3}=\left(q_{N_{d}+N_{n}+1}^{(k+1)}, \ldots, q_{N}^{(k+1)}\right)^{\mathrm{T}}$ are unknown.

On the other hand, by collocation (22) at nodes $\left\{\mathbf{x}_{i}\right\}_{i=N_{d}+N_{n}+1}^{N} \subset \Gamma_{S}$, we obtain

$$
\begin{array}{r}
q_{i}^{(k+1)}+c u_{i}^{(k+1)}=q_{i}^{(k)}+c u_{i}^{(k)}-\omega R\left(u_{i}^{(k)}, q_{i}^{(k)}\right), \\
i=N_{d}+N_{n}+1, \ldots, N .
\end{array}
$$

It is represented in matrix form as follows:

$$
\mathbf{Q}_{3}+c \mathbf{U}_{3}=\overline{\mathbf{F}}
$$

where $\overline{\mathbf{F}}=\left(f_{N_{d}+N_{n}+1}, f_{N_{d}+N_{n}+2}, \ldots, f_{N}\right)^{\mathrm{T}}$ with $f_{i}=q_{i}^{(k)}+$ $c u_{i}^{(k)}-\omega R\left(u_{i}^{(k)}, q_{i}^{(k)}\right)$.

Equations (29) and (31) contain a set of coupled $N+N_{s}$ equations together for $N+N_{s}$ unknowns $\mathbf{Q}_{1}, \mathbf{U}_{2}, \mathbf{U}_{3}$, and $\mathbf{Q}_{3}$. Therefore, if these $N+N_{s}$ simultaneous equations are solved directly, a considerable computational may be wasted. In the following, an indirect approach will be given.

From (31), we have

$$
\mathbf{Q}_{3}=\overline{\mathbf{F}}-c \mathbf{U}_{3} \text {. }
$$

Inserting (32) into (29), and then taking all unknown variables to the left-hand side while all known variables to the right-hand side, we can deduce the following $N \times N$ linear system of equations

$$
\begin{gathered}
{\left[\begin{array}{lll}
-\mathbf{G}_{11} & \mathbf{H}_{12} & \mathbf{H}_{13}+c \mathbf{G}_{13} \\
-\mathbf{G}_{21} & \mathbf{H}_{22} & \mathbf{H}_{23}+c \mathbf{G}_{23} \\
-\mathbf{G}_{31} & \mathbf{H}_{32} & \mathbf{H}_{33}+c \mathbf{G}_{33}
\end{array}\right]\left[\begin{array}{l}
\mathbf{Q}_{1} \\
\mathbf{U}_{2} \\
\mathbf{U}_{3}
\end{array}\right]} \\
=\left[\begin{array}{lll}
-\mathbf{H}_{11} & \mathbf{G}_{12} & \mathbf{G}_{13} \\
-\mathbf{H}_{21} & \mathbf{G}_{22} & \mathbf{G}_{23} \\
-\mathbf{H}_{31} & \mathbf{G}_{32} & \mathbf{G}_{33}
\end{array}\right]\left[\begin{array}{l}
\overline{\mathbf{U}}_{1} \\
\overline{\mathbf{Q}}_{2} \\
\overline{\mathbf{F}}
\end{array}\right] .
\end{gathered}
$$

Equation (33) contains $N$ equations and is now first solved for $N$ unknowns $\mathbf{Q}_{1}, \mathbf{U}_{2}$, and $\mathbf{U}_{3}$; then, substituting $\mathbf{U}_{3}$ into (32), the unknown $\mathbf{Q}_{3}$ can be solved easily.

3.5. Iterative Algorithm. Iterative algorithm of the current meshfree method for Signorini problems (10)-(13) is concluded in this subsection.

At each iteration, (33) needs to be solved. To improve the computational efficiency, an effectual approach for solving (33) would consist of computing the matrix

$$
\mathbf{A}=\left[\begin{array}{lll}
-\mathbf{G}_{11} & \mathbf{H}_{12} & \mathbf{H}_{13}+c \mathbf{G}_{13} \\
-\mathbf{G}_{21} & \mathbf{H}_{22} & \mathbf{H}_{23}+c \mathbf{G}_{23} \\
-\mathbf{G}_{31} & \mathbf{H}_{32} & \mathbf{H}_{33}+c \mathbf{G}_{33}
\end{array}\right]^{-1}\left[\begin{array}{lll}
-\mathbf{H}_{11} & \mathbf{G}_{12} & \mathbf{G}_{13} \\
-\mathbf{H}_{21} & \mathbf{G}_{22} & \mathbf{G}_{23} \\
-\mathbf{H}_{31} & \mathbf{G}_{32} & \mathbf{G}_{33}
\end{array}\right]
$$

at the initial stage and then computing the product $\mathbf{A b}$ at each iteration, where $\mathbf{b}=\left[\overline{\mathbf{U}}_{1}^{\mathrm{T}}, \overline{\mathbf{Q}}_{2}^{\mathrm{T}}, \overline{\mathbf{F}}\right]^{\mathrm{T}}$. Therefore, the system matrices in the method only have to be computed once.

Algorithm 2. Choose tolerance $\tau>0$ and $N$ nodes on the boundary $\Gamma$, and set $k=0$.

(1) Assume initially that the boundary condition on the Signorini boundary $\Gamma_{S}$ is $u^{(0)}=h$; then obtain the normal derivative $q^{(0)}$ on $\Gamma_{S}$ and coefficient matrices 
$\mathbf{H}$ and $\mathbf{G}$ by solving the following boundary value problem:

$$
\begin{aligned}
& \Delta u^{(0)}=0, \quad \text { in } \Omega, \\
& u^{(0)}=\bar{u}, \quad \text { on } \Gamma_{D}, \\
& q^{(0)}=\bar{q}, \quad \text { on } \Gamma_{N}, \\
& u^{(0)}=h, \quad \text { on } \Gamma_{S} .
\end{aligned}
$$

(2) Compute $\mathbf{A}$ and $f_{i}=q_{i}^{(0)}+c u_{i}^{(0)}-\omega R\left(u_{i}^{(0)}, q_{i}^{(0)}\right)$, and set $\overline{\mathbf{F}}=\left(f_{N_{d}+N_{n}+1}, f_{N_{d}+N_{n}+2}, \ldots, f_{N}\right)$ and $\mathbf{b}=$ $\left[\overline{\mathbf{U}}_{1}^{\mathrm{T}}, \overline{\mathbf{Q}}_{2}^{\mathrm{T}}, \overline{\mathbf{F}}\right]^{\mathrm{T}}$.

(3) Obtain $\mathbf{Q}_{1}^{(k+1)}, \mathbf{U}_{2}^{(k+1)}$, and $\mathbf{U}_{3}^{(k+1)}$ by computing $\mathbf{A b}$, and then obtain $\mathbf{Q}_{3}^{(k+1)}$ by $\mathbf{Q}_{3}^{(k+1)}=\overline{\mathbf{F}}-c \mathbf{U}_{3}^{(k+1)}$.

(4) If $\left\|\mathbf{U}_{3}^{(k+1)}-\mathbf{U}_{3}^{(k)}\right\|_{\Gamma_{S}} \leq \tau\left\|\mathbf{U}_{3}^{(k+1)}\right\|_{\Gamma_{S}}$, stop the algorithm and return $\mathbf{Q}_{1}^{(k+1)}, \mathbf{U}_{2}^{(k+1)}, \mathbf{U}_{3}^{(k+1)}$, and $\mathbf{Q}_{3}^{(k+1)}$.

(5) Otherwise, compute $f_{i}=q_{i}^{(k+1)}+$ $c u_{i}^{(k+1)}-\omega R\left(u_{i}^{(k+1)}, q_{i}^{(k+1)}\right)$, and set $\overline{\mathbf{F}}=$ $\left(f_{N_{d}+N_{n}+1}, f_{N_{d}+N_{n}+2}, \ldots, f_{N}\right)$ and $\mathbf{b}=\left[\overline{\mathbf{U}}_{1}^{\mathrm{T}}, \overline{\mathbf{Q}}_{2}^{\mathrm{T}}, \overline{\mathbf{F}}\right]^{\mathrm{T}}$. Update $k$ to $k+1$, and go to 3 .

\section{Convergence Analysis}

For mesh-based numerical methods, the convergence of some algorithms has been established for Signorini problems $[1,9,26,27]$. In this section, the convergence of the presented meshfree method is established. For the sake of proving the main convergence theorem, we need the following lemmas.

Lemma 3. Let $\left(u^{*}, q^{*}\right)$ and $(u, q)$ be the solutions of the Signorini problem (10)-(13) and the fixed point equation (17), respectively. Then

$$
\left\langle R(u, q)-c(u-h), q-q^{*}-R(u, q)\right\rangle_{\Gamma_{S}} \geq 0,
$$

where the duality $\langle\cdot, \cdot\rangle_{\Gamma_{S}}$ is defined for functions $w$ and $v$ as $\langle w, v\rangle_{\Gamma_{S}}=\int_{\Gamma_{S}} w v \mathrm{~d} \Gamma$.

Proof. If $(u, q)$ satisfies $u=h$ and $q \geq g$ on the Signorini boundary $\Gamma_{S}$, then for any positive constant $c$ we get $(q-g)-$ $c(u-h) \geq 0$; thus

$$
(q-g)-c(u-h)=[(q-g)-c(u-h)]_{+} .
$$

Otherwise, if $u \geq h$ and $q=g$ on $\Gamma_{S}$, then $(q-g)-c(u-h) \leq 0$; thus

$$
(q-g)-c(u-h) \leq[(q-g)-c(u-h)]_{+} .
$$

Consequently, using (19), we have

$$
R(u, q)-c(u-h) \leq 0 .
$$

On the other hand, using $q^{*}-g \geq 0$ yields

$$
[(q-g)-c(u-h)]_{+}-\left(q^{*}-g\right) \leq 0 .
$$

Then using (19) again leads to

$$
q-q^{*}-R(u, q) \leq 0 .
$$

Finally, the proof is completed via collecting (39) and (41).

Lemma 4. Let $\left(u^{*}, q^{*}\right)$ and $(u, q)$ be the solutions of the Signorini problem (10)-(13) and the boundary value problem (18), respectively. Then

$$
\left\langle q-q^{*}+c\left(u-u^{*}\right), R(u, q)\right\rangle_{\Gamma_{S}} \geq\|R(u, q)\|_{\Gamma_{S}}^{2} .
$$

Proof. From the Signorini boundary condition (13), we have $\left\langle u^{*}-h, q^{*}-g\right\rangle_{\Gamma_{S}}=0$; thus

$$
\begin{aligned}
\left\langle u^{*}-h, q-q^{*}\right\rangle_{\Gamma_{S}} & =\left\langle u^{*}-h,(q-g)-\left(q^{*}-g\right)\right\rangle_{\Gamma_{S}} \\
& =\left\langle u^{*}-h, q-g\right\rangle_{\Gamma_{S}} \geq 0 .
\end{aligned}
$$

According to (17), we have $q=g+[(q-g)-c(u-h)]_{+}$. Inserting this equation into (43) yields

$$
\left\langle u^{*}-h, g+[(q-g)-c(u-h)]_{+}-q^{*}\right\rangle_{\Gamma_{S}} \geq 0 .
$$

Then using (19) leads to

$$
\left\langle u^{*}-h, q-q^{*}-R(u, q)\right\rangle_{\Gamma_{S}} \geq 0 .
$$

Thus by applying Lemma 3 we obtain

$$
\left\langle R(u, q)-c(u-h)+c\left(u^{*}-h\right), q-q^{*}-R(u, q)\right\rangle_{\Gamma_{S}} \geq 0 .
$$

Namely,

$$
\begin{aligned}
& \left\langle R(u, q), q-q^{*}\right\rangle_{\Gamma_{S}}-\langle R(u, q), R(u, q)\rangle_{\Gamma_{S}} \\
& \quad-\left\langle c\left(u-u^{*}\right), q-q^{*}\right\rangle_{\Gamma_{S}}+\left\langle c\left(u-u^{*}\right), R(u, q)\right\rangle_{\Gamma_{S}} \geq 0 ;
\end{aligned}
$$

hence

$$
\begin{aligned}
& \left\langle q-q^{*}+c\left(u-u^{*}\right), R(u, q)\right\rangle_{\Gamma_{S}} \\
& \geq\left\langle q-q^{*}, c\left(u-u^{*}\right)\right\rangle_{\Gamma_{S}}+\|R(u, q)\|_{\Gamma_{S}}^{2} .
\end{aligned}
$$

Besides, Green's formula implies that

$$
\left\langle q-q^{*}, u-u^{*}\right\rangle_{\Gamma_{S}}=\left\|\nabla\left(u-u^{*}\right)\right\|_{L^{2}(\Omega)}^{2} \geq 0 .
$$

Finally, substituting this inequality into (48) ends the proof.

Theorem 5. The approximate solution $\left(u^{(k)}, q^{(k)}\right)$ obtained from Algorithm 2 converges to the unique solution $\left(u^{*}, q^{*}\right)$ of the Signorini problem (10)-(13). 
Proof. Applying (22) provides

$$
\begin{aligned}
\| & \left(q^{(k+1)}-q^{*}\right)+c\left(u^{(k+1)}-u^{*}\right) \|_{\Gamma_{S}}^{2} \\
= & \left\|\left(q^{(k)}-q^{*}\right)+c\left(u^{(k)}-u^{*}\right)-\omega R\left(u^{(k)}, q^{(k)}\right)\right\|_{\Gamma_{S}}^{2} \\
= & \left\|\left(q^{(k)}-q^{*}\right)+c\left(u^{(k)}-u^{*}\right)\right\|_{\Gamma_{S}}^{2}+\omega^{2}\left\|R\left(u^{(k)}, q^{(k)}\right)\right\|_{\Gamma_{S}}^{2} \\
& -2 \omega\left\langle\left(q^{(k)}-q^{*}\right)+c\left(u^{(k)}-u^{*}\right), R\left(u^{(k)}, q^{(k)}\right)\right\rangle_{\Gamma_{S}} .
\end{aligned}
$$

Then, according to Lemma 4, we obtain

$$
\begin{gathered}
\left\|\left(q^{(k+1)}-q^{*}\right)+c\left(u^{(k+1)}-u^{*}\right)\right\|_{\Gamma_{S}}^{2} \\
\leq\left\|\left(q^{(k)}-q^{*}\right)+c\left(u^{(k)}-u^{*}\right)\right\|_{\Gamma_{S}}^{2} \\
\quad-\omega(2-\omega)\left\|R\left(u^{(k)}, q^{(k)}\right)\right\|_{\Gamma_{S}}^{2} .
\end{gathered}
$$

Hence,

$$
\begin{aligned}
& \sum_{k=0}^{\infty} \omega(2-\omega)\left\|R\left(u^{(k)}, q^{(k)}\right)\right\|_{\Gamma_{S}}^{2} \\
& \quad \leq\left\|\left(q^{(0)}-q^{*}\right)+c\left(u^{(0)}-u^{*}\right)\right\|_{\Gamma_{S}}^{2},
\end{aligned}
$$

which implies that $\lim _{k \rightarrow \infty} R\left(u^{(k)}, q^{(k)}\right)=0$.

Let $\left(u^{*}, q^{*}\right)$ be the cluster point of $\left\{\left(u^{(k)}, q^{(k)}\right)\right\}$ and the subsequence $\left\{\left(u^{\left(k_{1}\right)}, q^{\left(k_{1}\right)}\right)\right\}$ of the sequence $\left\{\left(u^{(k)}, q^{(k)}\right)\right\}$ converges to $\left(u^{*}, q^{*}\right)$. Since $R(u, q)$ is continuous, it follows that

$$
R\left(u^{*}, q^{*}\right)=\lim _{k \rightarrow \infty} R\left(u^{\left(k_{1}\right)}, q^{\left(k_{1}\right)}\right)=0,
$$

which implies that $\left(u^{*}, q^{*}\right)$ is a solution of the Signorini problem (10)-(13) by invoking Theorem 1 .

To prove that $\left(u^{*}, q^{*}\right)$ is the unique cluster point of the sequence $\left\{\left(u^{(k)}, q^{(k)}\right)\right\}$, we now assume that $\left(u^{* *}, q^{* *}\right)$ is another cluster point and let

$$
\delta=\left\|q^{* *}-q^{*}\right\|_{\Gamma_{S}}
$$

Since $\left(u^{*}, q^{*}\right)$ is the cluster point of $\left\{\left(u^{(k)}, q^{(k)}\right)\right\}$, there exists a positive integer $\bar{k}$ such that

$$
\left\|\left(q^{(\bar{k})}-q^{*}\right)+c\left(u^{(\bar{k})}-u^{*}\right)\right\|_{\Gamma_{S}} \leq \frac{\delta}{2} .
$$

Besides, using Green's formula yields

$$
\left\langle q^{(k)}-q^{*}, u^{(k)}-u^{*}\right\rangle_{\Gamma_{S}}=\left\|\nabla\left(u^{(k)}-u^{*}\right)\right\|_{L^{2}(\Omega)}^{2} \geq 0 .
$$

Thus

$$
\begin{aligned}
& \left\|\left(q^{(k)}-q^{*}\right)+c\left(u^{(k)}-u^{*}\right)\right\|_{\Gamma_{S}}^{2} \\
& =\left\|q^{(k)}-q^{*}\right\|_{\Gamma_{S}}^{2}+c^{2}\left\|u^{(k)}-u^{*}\right\|_{\Gamma_{S}}^{2} \\
& \quad+2 c\left\langle q^{(k)}-q^{*}, u^{(k)}-u^{*}\right\rangle_{\Gamma_{S}} \\
& \geq\left\|q^{(k)}-q^{*}\right\|_{\Gamma_{S}}^{2} .
\end{aligned}
$$

According to (51) we get

$$
\begin{aligned}
& \left\|\left(q^{(k+1)}-q^{*}\right)+c\left(u^{(k+1)}-u^{*}\right)\right\|_{\Gamma_{s}}^{2} \\
& \quad \leq\left\|\left(q^{(k)}-q^{*}\right)+c\left(u^{(k)}-u^{*}\right)\right\|_{\Gamma_{s}}^{2}
\end{aligned}
$$

hence for any $k>\bar{k}$,

$$
\begin{gathered}
\left\|q^{(k)}-q^{*}\right\|_{\Gamma_{S}} \leq\left\|\left(q^{(k)}-q^{*}\right)+c\left(u^{(k)}-u^{*}\right)\right\|_{\Gamma_{S}} \\
\leq\left\|\left(q^{(\bar{k})}-q^{*}\right)+c\left(u^{(\bar{k})}-u^{*}\right)\right\|_{\Gamma_{S}} \leq \frac{\delta}{2} .
\end{gathered}
$$

Consequently,

$$
\left\|q^{(k)}-q^{* *}\right\|_{\Gamma_{S}} \geq\left\|q^{* *}-q^{*}\right\|_{\Gamma_{S}}-\left\|q^{(k)}-q^{*}\right\|_{\Gamma_{S}} \geq \frac{\delta}{2}
$$

which implies that $\lim _{k \rightarrow \infty} q^{(k)} \neq q^{* *}$. As a result, the sequence $\left\{\left(u^{(k)}, q^{(k)}\right)\right\}$ has exactly one cluster point and therefore,

$$
\lim _{k \rightarrow \infty}\left(u^{(k)}, q^{(k)}\right)=\left(u^{*}, q^{*}\right)
$$

which completes the proof.

\section{Numerical Examples}

5.1. Validations. A Signorini problem with known analytical solutions is first considered to verify the efficiency of the current meshfree method.

This example embraces a Signorini problem on the annular domain $\Omega=\left\{\mathbf{x} \in \mathbb{R}^{2}: a<|\mathbf{x}|<b\right\}$. The analytical solution is given by the following complex function [8]:

$$
u\left(x_{1}, x_{2}\right)=\operatorname{Im} w\left(x_{1}+\mathrm{i} x_{2}\right)^{3},
$$


where

$$
\begin{aligned}
& w\left(x_{1}+\mathrm{i} x_{2}\right)=\left(\frac{1}{2} \sqrt{\left(\frac{x_{1}^{2}-x_{2}^{2}}{r^{2}}\right)^{2}+\frac{1}{4}\left(\frac{r^{2}}{a^{2}}-\frac{a^{2}}{r^{2}}\right)^{2}}\right. \\
&+\frac{\left.x_{1}^{2}-x_{2}^{2}\left(\frac{r^{2}}{a^{2}}+\frac{a^{2}}{r^{2}}\right)\right)^{1 / 2} \operatorname{sign} x_{1}}{+} \\
&+\left(\frac{1}{2} \sqrt{\left(\frac{x_{1}^{2}-x_{2}^{2}}{r^{2}}\right)^{2}+\frac{1}{4}\left(\frac{r^{2}}{a^{2}}-\frac{a^{2}}{r^{2}}\right)^{2}}\right. \\
&\left.-\frac{x_{1}^{2}-x_{2}^{2}}{4 r^{2}}\left(\frac{r^{2}}{a^{2}}+\frac{a^{2}}{r^{2}}\right)\right)^{1 / 2} \operatorname{sign} x_{2},
\end{aligned}
$$

with $r^{2}=x_{1}^{2}+x_{2}^{2}$.

Dirichlet conditions corresponding to the analytical solution are prescribed on $\Gamma_{D}=\left\{\mathbf{x} \in \mathbb{R}^{2}:|\mathbf{x}|=b\right\}$. Signorini boundary conditions on $\Gamma_{S}=\left\{\mathbf{x} \in \mathbb{R}^{2}:|\mathbf{x}|=a\right\}$ are

$$
u \geq h, \quad q \geq g, \quad(u-h)(q-g)=0,
$$

where

$$
\begin{gathered}
h\left(x_{1}, x_{2}\right)=\min \left(0, u\left(x_{1}, x_{2}\right)\right), \\
g\left(x_{1}, x_{2}\right)= \begin{cases}-\frac{6}{a}, & x_{2}>0, \\
-\frac{6}{a^{5}}\left(x_{1}^{2}-x_{2}^{2}\right)^{2}, & x_{2} \leq 0, x_{2} \geq-\left|x_{1}\right|, \\
0, & x_{2}<-\left|x_{1}\right| .\end{cases}
\end{gathered}
$$

The analytical solution on $\Gamma_{S}$ is

$$
\begin{gathered}
u\left(x_{1}, x_{2}\right)=-\sqrt{\max \left(0, \frac{x_{2}^{2}-x_{1}^{2}}{a^{2}}\right)^{3}} \operatorname{sign} x_{2}, \\
q\left(x_{1}, x_{2}\right)=-\frac{6}{a^{3}} \sqrt{\max \left(0, \frac{x_{1}^{2}-x_{2}^{2}}{a^{2}}\right)}\left|x_{1}\right| x_{2} .
\end{gathered}
$$

In the calculations, we take $a=0.1$ and $b=0.25$ and use the parameterizations $t \rightarrow(a \cos 2 \pi t,-a \sin 2 \pi t)$ and $t \rightarrow$ $(b \cos 2 \pi t, b \sin 2 \pi t)$. The analytical and numerical solutions for the potential $u$ and its normal derivative $q$ are exhibited in Figure 1. In this analysis, we used 64 boundary nodes. It can be found that the numerical results agree well with the analytical solutions.

In order to show the convergence of our method, a norm error is defined as

$$
e(u)=\frac{1}{N_{s}} \sqrt{\sum_{i=1}^{N_{s}}\left(u^{(i)}-u_{h}^{(i)}\right)^{2}}
$$

where $u^{(i)}$ and $u_{h}^{(i)}$ are analytical and numerical solutions, respectively.

In BIEs-based meshfree method, it has been observed that the positions of the boundary nodes on each cell can affect the accuracy of numerical solutions. In this research, we also studied the influence of the positions of the boundary nodes on the accuracy of the developed meshfree method. The position of the boundary node on a cell is determined by a parameter $\alpha, 0 \leq \alpha \leq 1$, using the following equation:

$$
\mathbf{x}_{P}=\alpha \mathbf{x}_{L}+(1-\alpha) \mathbf{x}_{R}
$$

where $\mathbf{x}_{P}$ is the boundary node and $\mathbf{x}_{L}$ and $\mathbf{x}_{R}$ are the left and the right end points of a cell, respectively. Obviously, the boundary node is at the center of the cell when $\alpha=0.5$ and at the left and the right ends of the cell when $\alpha=0$ and $\alpha=1$, respectively.

Figure 2 gives the log-log plot of errors with respect to the number of boundary nodes. Five kinds of nodal arrangement of $32,64,128,256$, and 512 boundary nodes are used. It can be seen that our method yields very accurate results and has high convergence rate.

The error results for various values of $\alpha$ are shown in Figure 2. We can see that the computational accuracy is influenced by $\alpha$. However, comparing with the results in the BNM [10], the accuracy of our method is much less sensitive to the relative position of the boundary node in a cell than that of the BNM. In all cases, even when the boundary nodes are close to the ends of the cells, our method has yielded satisfactory results.

\subsection{Applications}

5.2.1. The Shallow Dam Problem. In this example, we consider the groundwater flow problem related to percolation in gently sloping beaches (see Figure 3). This problem is known as the shallow dam problem [2]. It concerns flow through a porous medium (such as sand) where the free boundary between the saturated region and dry region is close to the upper surface of the medium, which is supposed to be nearly horizontal. Because the domain of interest is the saturated region, the number and positions of the separation points need to be determined.

A mathematical model for the shallow dam problem can be formulated as the following Signorini problem:

$$
\begin{gathered}
\Delta u=0, \quad \text { in } \Omega=\left\{\left(x_{1}, x_{2}\right): 0 \leq x_{1} \leq 1,0 \leq x_{2} \leq 1\right\}, \\
u=0, \quad \text { on } \Gamma_{D}=\left\{\left(x_{1}, x_{2}\right): x_{1}=0,0 \leq x_{2} \leq 1\right\}, \\
\frac{\partial u}{\partial x_{2}}=0, \quad \text { on } \Gamma_{N}=\left\{\left(x_{1}, x_{2}\right): 0 \leq x_{1} \leq 1, x_{2}=0\right\}, \\
u=\mathrm{G}(1), \quad \text { on } \Gamma_{D}=\left\{\left(x_{1}, x_{2}\right): x_{1}=1,0 \leq x_{2} \leq 1\right\}, \\
u \leq \mathrm{G}\left(x_{1}\right), \quad \frac{\partial u}{\partial x_{2}} \leq 0, \quad\left(u-\mathrm{G}\left(x_{1}\right)\right) \frac{\partial u}{\partial x_{2}}=0, \\
\text { on } \Gamma_{S}=\left\{\left(x_{1}, x_{2}\right): 0 \leq x_{1} \leq 1, x_{2}=1\right\},
\end{gathered}
$$




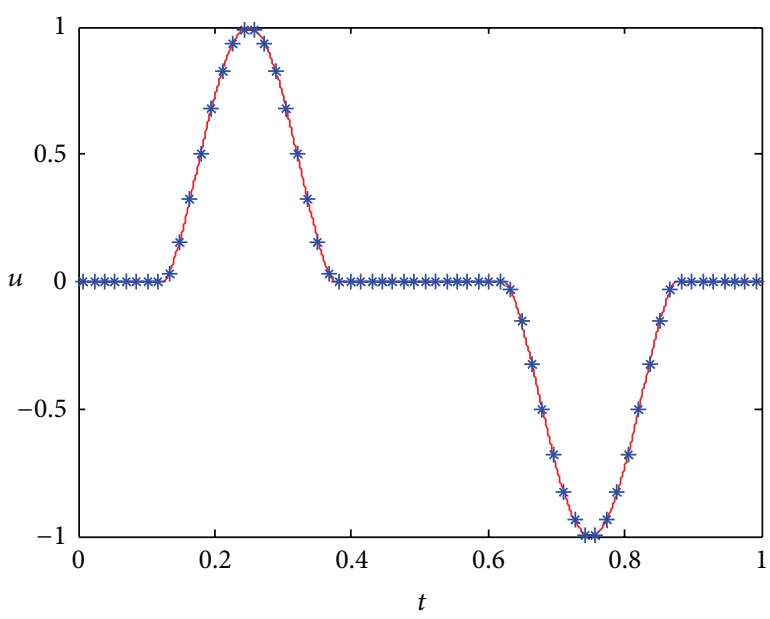

* Analytical solution

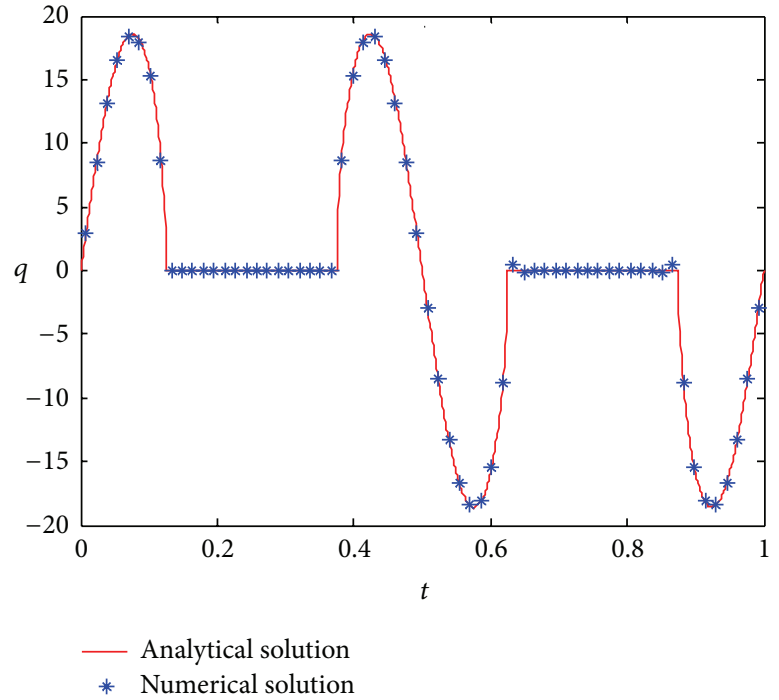

(b)

FIgURE 1: Results of (a) $u$ and (b) $q$ along $\Gamma_{S}$.

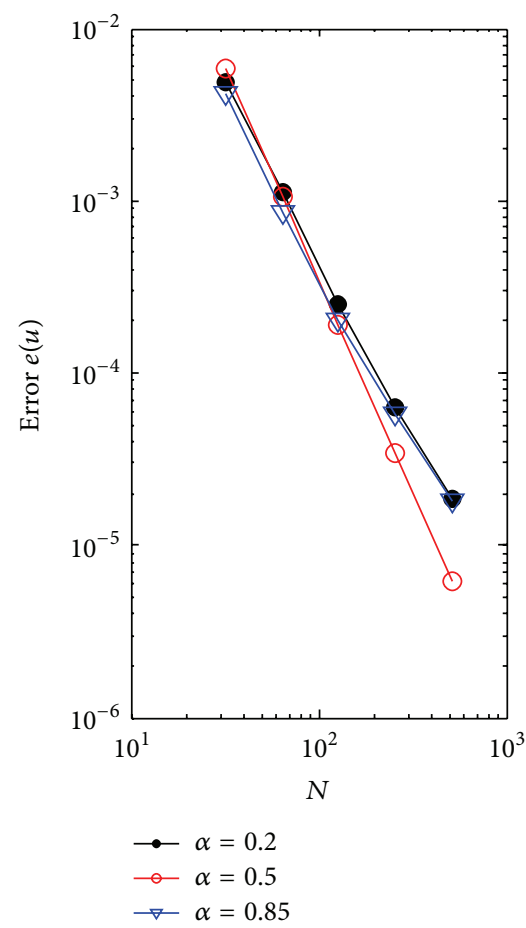

(a)

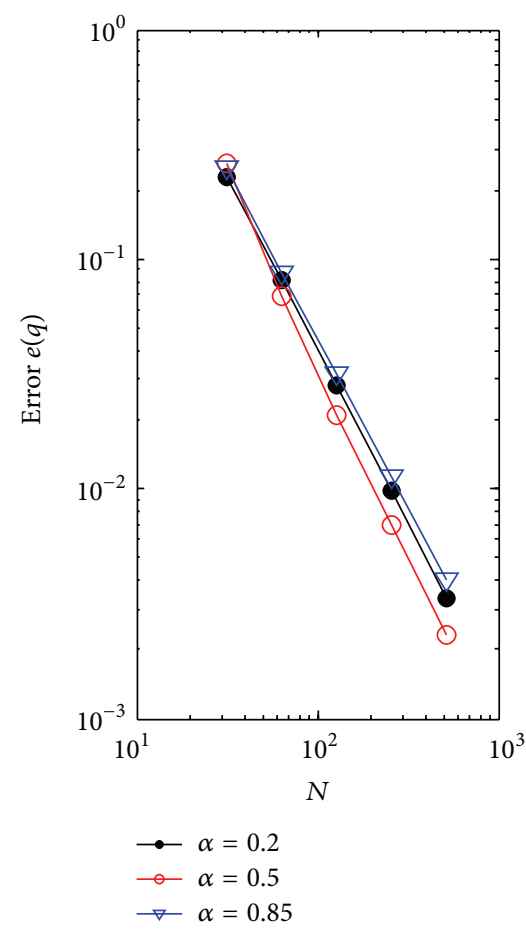

(b)

FIgURE 2: Convergence of (a) $u$ and (b) $q$.

where $G\left(x_{1}\right)$ is a known function representing the surface profile. Obviously, the number and positions of the separation points depend on $\mathrm{G}\left(x_{1}\right)$.

Figures 4, 5, and 6 plot the numerical results obtained with three different surface profiles given by

$$
\begin{gathered}
\mathrm{G}_{1}\left(x_{1}\right)=\left(\frac{1}{2}-x_{1}\right)\left(1-x_{1}\right)-x_{1}, \\
\mathrm{G}_{2}\left(x_{1}\right)=\left(\frac{1}{2}-\frac{5}{2} x_{1}\right)\left(1-\frac{5}{3} x_{1}\right)\left(1-x_{1}\right)-x_{1}, \\
\mathrm{G}_{3}\left(x_{1}\right)=\sin \left(12 x_{1}\right)-2 .
\end{gathered}
$$


TABLE 1: Numbers of iterations for the first profile $\mathrm{G}_{1}\left(x_{1}\right)$ and $\tau=10^{-6}$.

\begin{tabular}{lccccccc}
\hline$N$ & 28 & 48 & 64 & 84 & 128 & 512 & 1024 \\
\hline Our method & 25 & 25 & 24 & 24 & 25 & 25 \\
The MFS & 72 & 119 & 893 & 946 & & \\
\hline
\end{tabular}

TABle 2: Numbers of iterations for the first profile $G_{1}\left(x_{1}\right)$ and $N=64$.

\begin{tabular}{lccccc}
\hline$\tau$ & $10^{-2}$ & $10^{-3}$ & $10^{-4}$ & $10^{-5}$ & $10^{-6}$ \\
\hline Our method & 5 & 10 & 14 & 19 & 24 \\
The MFS & 77 & 84 & 270 & 345 & 893 \\
\hline
\end{tabular}

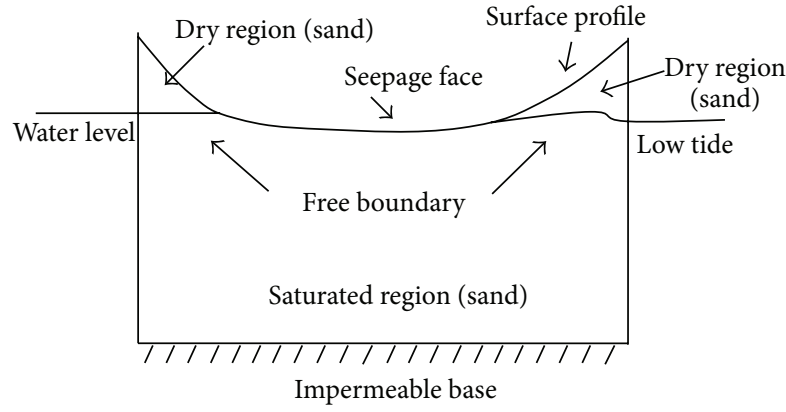

FIgURE 3: Schematic view of the shallow dam problem.

In this analysis, we used 21 boundary nodes on each side of the square. Although no analytical solutions exist for such a complex problem, it can be found that these graphs are indistinguishable from those given in [1-3].

To study the convergence of our method, we solve the problem via choosing various numbers of boundary nodes $(N)$ and various values of the tolerance $(\tau)$. The numerical results for the first profile $\mathrm{G}_{1}\left(x_{1}\right)$ are compared to those obtained using the method of fundamental solutions (MFS) [3]. Table 1 presents the numbers of iteration for various values of $N$ when keeping $\tau=10^{-6}$ fixed, while Table 2 presents the numbers of iteration for various values of $\tau$ when $N=64$ is kept fixed. We can observe that the numbers of iterations of our method are little influenced by the number of boundary nodes and are less than those of the MFS for both tests. Therefore, the convergence of our method is faster than that of the MFS.

Besides, we tabulate in Table 3 the calculated values of $u\left(x_{1}\right)$ on $\Gamma_{S}$ near the separation point. Recall that the separation point is the point where we have a change from conditions $u<\mathrm{G}\left(x_{1}\right)$ and $\partial u /\left(\partial x_{2}\right)=0$ to conditions $u=\mathrm{G}\left(x_{1}\right)$ and $\partial u / \partial x_{2}<0$ or vice versa. We can find that the values of $u\left(x_{1}\right)$ converge with $N$. On the section corresponding to the seepage face, the solution is in good agreement with the values of $\mathrm{G}_{1}\left(x_{1}\right)$.

5.2.2. The Electropainting Problem. The current meshfree method is also used to simulate the well-known electropainting problem [1]. This problem describes the coating process of metal surface with paint. It is very important for

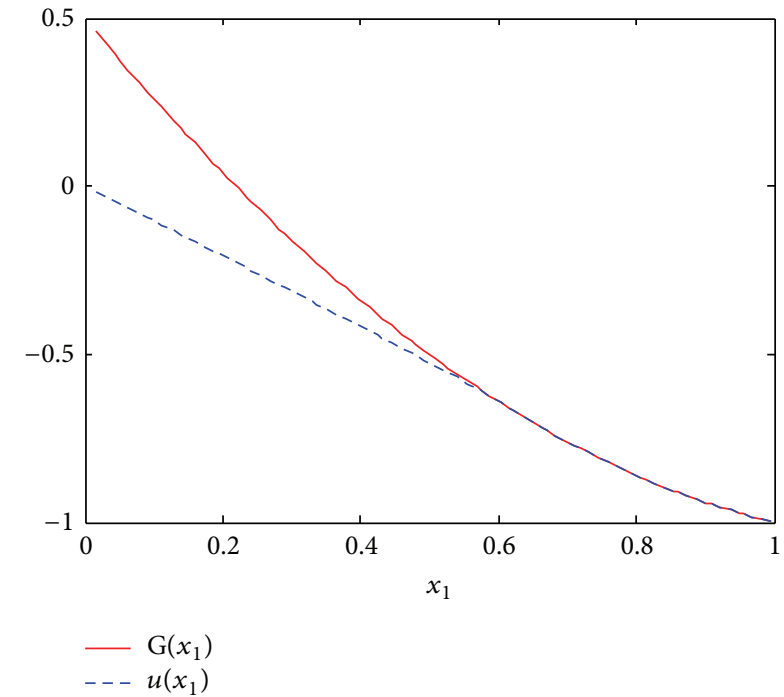

FIGURE 4: Approximate solution of the shallow dam problem with $\mathrm{G}_{1}\left(x_{1}\right)$.

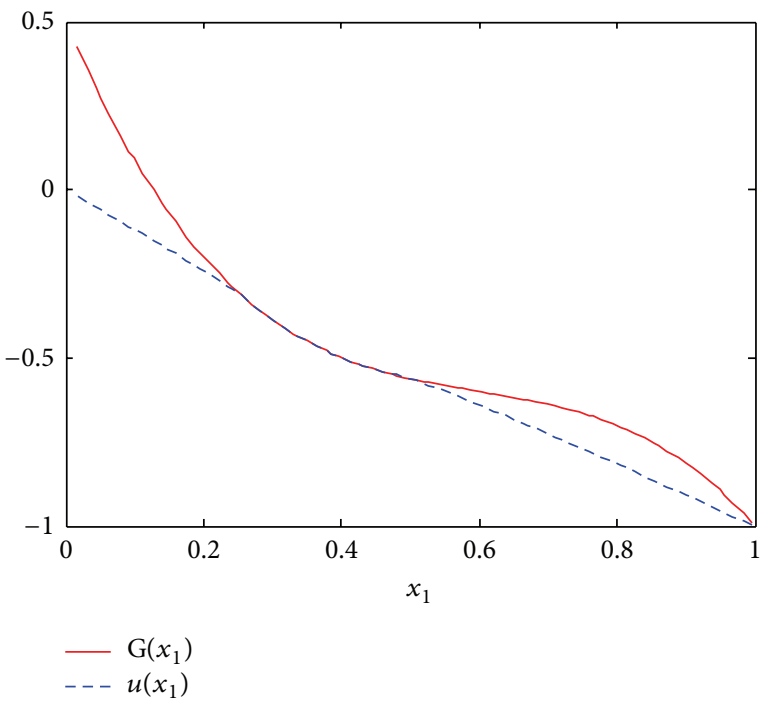

FIGURE 5: Approximate solution of the shallow dam problem with $\mathrm{G}_{2}\left(x_{1}\right)$. 
TABLE 3: Convergence with $N$ of the solution near the separation point for the first profile $\mathrm{G}_{1}\left(x_{1}\right)$ and $\tau=10^{-6}$.

\begin{tabular}{ccccccccc}
\hline$x_{1}$ & 0.6000 & 0.6500 & 0.7000 & 0.7500 & 0.8000 & 0.8500 & 0.9000 \\
\hline \multicolumn{2}{c}{$\mathrm{G}_{1}\left(x_{1}\right)$} & -0.6400 & -0.7025 & -0.7600 & -0.8125 & -0.8600 & -0.9025 & -0.9400 \\
\multirow{2}{*}{ The MFS $(N=84)$} & -0.6599 & -0.7148 & -0.7664 & -0.8125 & -0.8601 & -0.9027 & -0.9417 \\
\hline \multirow{2}{*}{ Our method } & $N=32$ & -0.6391 & -0.7022 & -0.7602 & -0.8131 & -0.8602 & -0.9017 & -0.9388 \\
& $N=64$ & -0.6395 & -0.7024 & -0.7600 & -0.8125 & -0.8600 & -0.9025 & -0.9400 \\
& $N=128$ & -0.6404 & -0.7025 & -0.7600 & -0.8125 & -0.8600 & -0.9025 & -0.9400 \\
& $N=512$ & -0.6400 & -0.7025 & -0.7600 & -0.8125 & -0.8600 & -0.9025 & -0.9400 \\
& $N=1024$ & -0.6400 & -0.7025 & -0.7600 & -0.8125 & -0.8600 & -0.9025 & -0.9400 \\
\hline \multicolumn{3}{c}{ Free boundary } \\
\hline
\end{tabular}

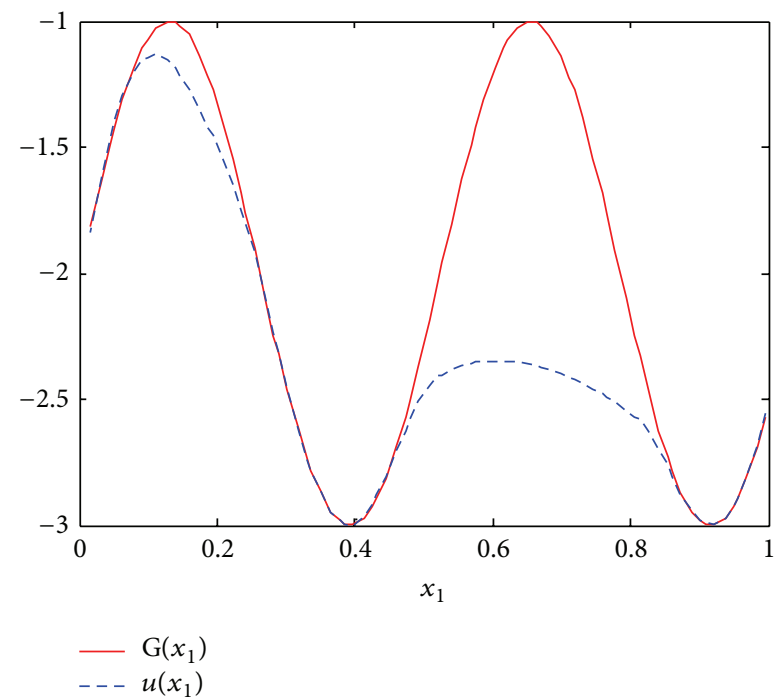

Figure 6: Approximate solution of the shallow dam problem with $\mathrm{G}_{3}\left(x_{1}\right)$.

the application of corrosion-protection paints to vehicle bodies in the motor manufacturing industry. The workpiece to be painted is immersed in a bath containing an electrolyte paint solution with charged ions. A potential difference is established between the workpiece and the edge of the bath. Therefore, the paint particles become charged and are deposited on the surface of the workpiece.

This problem can be formulated as a Signorini problem on a square domain $(-0.5,0.5) \times(0,1)$ for the electric potential $u$ in the paint solution. Due to the symmetry of the problem, only the right-hand side of the square domain is modeled as shown in Figure 7. Then, the electric potential $u$ satisfies

$$
\begin{gathered}
\Delta u=0, \quad \text { in } \Omega=\left\{\left(x_{1}, x_{2}\right): 0 \leq x_{1} \leq 0.5,0 \leq x_{2} \leq 1\right\}, \\
u=1, \quad \text { on } \Gamma_{D}=\left\{\left(x_{1}, x_{2}\right): 0 \leq x_{1} \leq 0.5, x_{2}=0\right\}, \\
q=0, \quad \text { on } \Gamma_{N}=\left\{\left(x_{1}, x_{2}\right): x_{1}=0,0 \leq x_{2} \leq 1\right\}, \\
u \geq 0, \quad q+\varepsilon \geq 0, \quad u(q+\varepsilon)=0, \quad \text { on } \Gamma_{S},
\end{gathered}
$$

where the known constant $\varepsilon$ is the critical cut-off current and is a property of the paint and the Signorini boundary $\Gamma_{S}=$

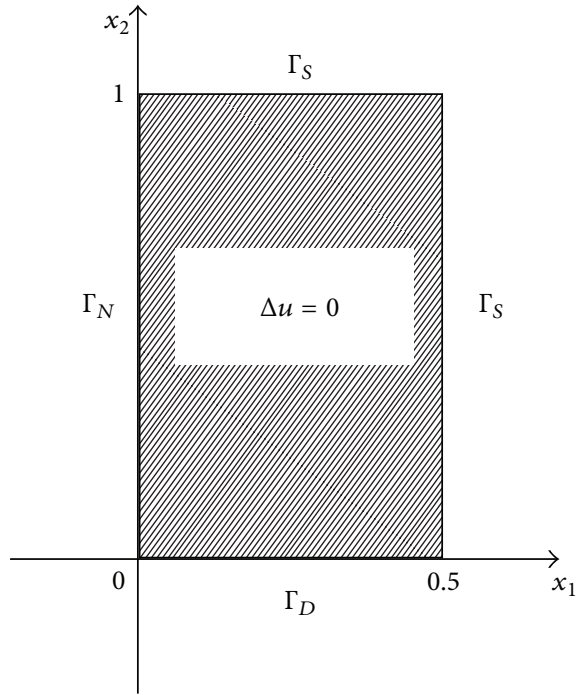

FIgURE 7: The right-hand side of the electropainting model.

$\left\{\left(x_{1}, x_{2}\right): x_{1}=0.5,0 \leq x_{2} \leq 1\right\} \cup\left\{\left(x_{1}, x_{2}\right): 0 \leq x_{1} \leq\right.$ $\left.0.5, x_{2}=1\right\}$ makes up the workpiece to be painted. On $\Gamma_{S}$, we have to decide which sections need to be painted $(u>0, q+$ $\varepsilon=0)$ and which need to be unpainted $(u=0, q+\varepsilon>0)$. The paint thickness is $u / \varepsilon$ and is very small. The solution of this problem contains the electropainting scope and the thickness distribution of the rectangle workpiece.

Figure 8 depicts the paint distribution over the domain, obtained for various values of $\varepsilon$. For the smaller values of $\varepsilon$, the workpiece is completely painted with the corner receiving the least amount of paint, as shown in Figure 8(a) where the results for $\varepsilon=0.4$ are plotted. If the value of $\varepsilon$ is increased, we observed that the paint film near the corner becomes thinner (Figure 8(b)) and eventually unpainted (Figure 8(c)). At an even higher value of $\varepsilon$, both the corner and the top boundary are unpainted (Figure $8(\mathrm{~d})$ ). Therefore, although there are no analytical solutions available in the literature to verify the current meshfree method for such a complex problem, the reliability of the method can be judged from the reasonable physical characteristics. Besides, it can be found that the results obtained by the current method are in very good agreement with those obtained using the FEM, the MFS, and the BEM $[1,3,4]$. 


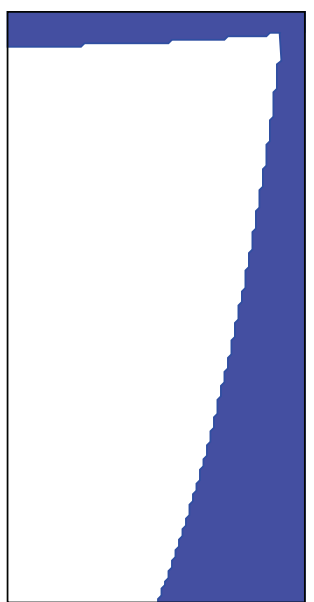

(a)

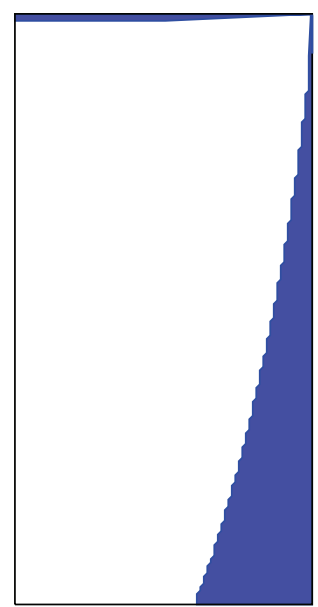

(b)

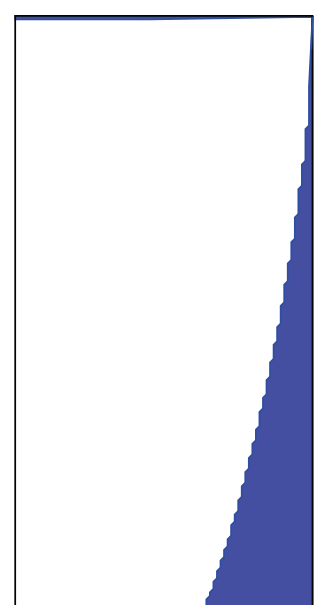

(c)

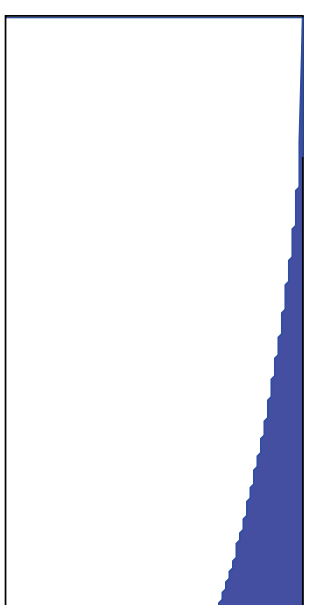

(d)

Figure 8: Paint distributions for (a) $\varepsilon=0.4$, (b) $\varepsilon=0.5$, (c) $\varepsilon=0.55$, and (d) $\varepsilon=0.7$.

\section{Conclusions}

In this study, we have developed a meshfree method based on BIEs for direction solution of Signorini problems. In this numerical method, boundary inequality constraints are incorporated into the iterative scheme naturally, and boundary conditions can be applied directly and easily. Besides, the current implementation only requires a nodal data structure on the bounding surface of a body whose dimension is one less than that of the domain itself. Moreover, this method is particularly suitable for Signorini problems since, in these, it is the Signorini boundary which is of prime interest. Therefore, the size of the linear system of equations resulting from the current method is much smaller and the required data manipulation is considerably reduced. Convergence for the proposed method has been proven theoretically. Some examples have been given and the numerical results are accurate and are in agreement with analytical solutions and previously reported numerical solutions.

Initial numerical results from the developed meshfree method, applied to Signorini problems, are encouraging. The numerical experiments indicate that this meshfree method provides monotonic convergence and high accuracy results. Besides, due to its considerable flexibility in mesh generation and relative ease in implementation, this method has tremendous potential for efficiently solving problems with complex domains to take the full advantages of the meshfree concept. Nevertheless, more research work is required.

\section{Conflict of Interests}

The authors declare that there is no conflict of interests regarding the publication of this paper.

\section{Acknowledgments}

This work was supported by National Natural Science Foundation of China under Grant no. 11101454, the Educational
Commission Foundation of Chongqing of China under Grant no. KJ130626, the Natural Science Foundation Project of CQ CSTC under Grant no. cstc2013jcyjA30001, and the Program of Chongqing Innovation Team Project in University under Grant no. KJTD201308.

\section{References}

[1] J. M. Aitchison and M. W. Poole, "A numerical algorithm for the solution of Signorini problems," Journal of Computational and Applied Mathematics, vol. 94, no. 1, pp. 55-67, 1998.

[2] J. M. Aitchison, C. M. Elliott, and J. R. Ockendon, "Percolation in gently sloping beaches," IMA Journal of Applied Mathematics, vol. 30, no. 3, pp. 269-287, 1983.

[3] A. Poullikkas, A. Karageorghis, and G. Georgiou, "The method of fundamental solutions for Signorini problems," IMA Journal of Numerical Analysis, vol. 18, no. 2, pp. 273-285, 1998.

[4] J. M. Aitchison, A. A. Lacey, and M. Shillor, "A model for an electropaint process," IMA Journal of Applied Mathematics, vol. 33, no. 1, pp. 17-31, 1984.

[5] R. Glowinski, Numerical Methods for Nonlinear Variational Problems, Springer, New York, NY, USA, 1984.

[6] S. D. Howison, J. D. Morgan, and J. R. Ockendon, "A class of codimension-two free boundary problems," SIAM Reviews, vol. 39, no. 2, pp. 221-253, 1997.

[7] A. Karageorghis, "Numerical solution of a shallow dam problem by a boundary element method," Computer Methods in Applied Mechanics and Engineering, vol. 61, no. 3, pp. 265-276, 1987.

[8] W. Spann, "On the boundary element method for the Signorini problem of the Laplacian," Numerische Mathematik, vol. 65, no. 3, pp. 337-356, 1993.

[9] S. Zhang and J. Zhu, "A projection iterative algorithm boundary element method for the Signorini problem," Engineering Analysis with Boundary Elements, vol. 37, no. 1, pp. 176-181, 2013.

[10] S. Mukherjee and Y. X. Mukherjee, Boundary Methods: Elements, Contours, and Nodes, vol. 187, CRC Press, Boca Raton, Fla, USA, 2005.

[11] G. R. Liu, Meshfree Methods: Moving beyond the Finite Element Method, CRC Press, Boca Raton, Fla, USA, 2nd edition, 2009. 
[12] M. Jamil and E. Y. K. Ng, "Evaluation of meshless radial basis collocation method (RBCM) for heterogeneous conduction and simulation of temperature inside the biological tissues," International Journal of Thermal Sciences, vol. 68, pp. 42-52, 2013.

[13] A. Khayyer, H. Gotoh, and S. D. Shao, "Corrected Incompressible SPH method for accurate water-surface tracking in breaking waves," Coastal Engineering, vol. 55, no. 3, pp. 236-250, 2008.

[14] T. W. H. Sheu, C. Chiao, and C. Huang, "Development of a particle interaction kernel function in MPS method for simulating incompressible free surface flow," Journal of Applied Mathematics, vol. 2011, Article ID 793653, 16 pages, 2011.

[15] Y. Xie Mukherjee and S. Mukherjee, "The boundary node method for potential problems," International Journal for Numerical Methods in Engineering, vol. 40, no. 5, pp. 797-815, 1997.

[16] K. M. Liew, Y. Cheng, and S. Kitipornchai, "Boundary elementfree method (BEFM) and its application to two-dimensional elasticity problems," International Journal for Numerical Methods in Engineering, vol. 65, no. 8, pp. 1310-1332, 2006.

[17] R. Hongping, C. Yumin, and Z. Wu, "An interpolating boundary element-free method (IBEFM) for elasticity problems," Science China, vol. 53, no. 4, pp. 758-766, 2010.

[18] X. Li and J. Zhu, "A Galerkin boundary node method and its convergence analysis," Journal of Computational and Applied Mathematics, vol. 230, no. 1, pp. 314-328, 2009.

[19] X. Li, "The meshless Galerkin boundary node method for Stokes problems in three dimensions," International Journal for Numerical Methods in Engineering, vol. 88, no. 5, pp. 442-472, 2011.

[20] X. Li, "Numerical solution of solid mechanics problems using a boundary-only and truly meshless method," Mathematical Problems in Engineering, vol. 2012, Article ID 298903, 16 pages, 2012.

[21] Y. T. Gu and G. R. Liu, "A boundary point interpolation method for stress analysis of solids," Computational Mechanics, vol. 28, no. 1, pp. 47-54, 2002.

[22] G. R. Liu and Y. T. Gu, "Boundary meshfree methods based on the boundary point interpolation methods," Engineering Analysis with Boundary Elements, vol. 28, no. 5, pp. 475-487, 2004.

[23] Y. T. Gu and G. R. Liu, "Hybrid boundary point interpolation methods and their coupling with the element free Galerkin method," Engineering Analysis with Boundary Elements, vol. 27, no. 9, pp. 905-917, 2003.

[24] G. R. Liu and Y. T. Gu, "A point interpolation method for two-dimensional solids," International Journal for Numerical Methods in Engineering, vol. 50, pp. 937-951, 2001.

[25] J. G. Wang and G. R. Liu, "A point interpolation meshless method based on radial basis functions," International Journal for Numerical Methods in Engineering, vol. 54, no. 11, pp. 16231648, 2002.

[26] M. Aslam Noor, "Some developments in general variational inequalities," Applied Mathematics and Computation, vol. 152, no. 1, pp. 199-277, 2004.

[27] J. Y. Han, N. H. Xiu, and H. D. Qi, Nonlinear Complementarity Theory and Algorithm, Shanghai Science and Technology Press, Shanghai, China, 2006.

[28] M. A. Noor, "Projection iterative methods for extended general variational inequalities," Journal of Applied Mathematics and Computing, vol. 32, no. 1, pp. 83-95, 2010. 


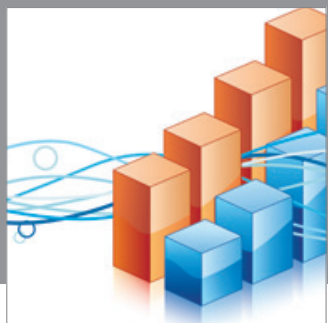

Advances in

Operations Research

mansans

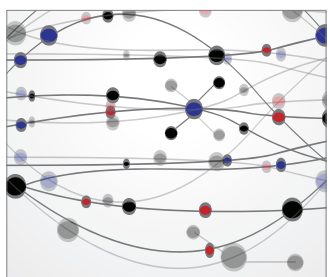

The Scientific World Journal
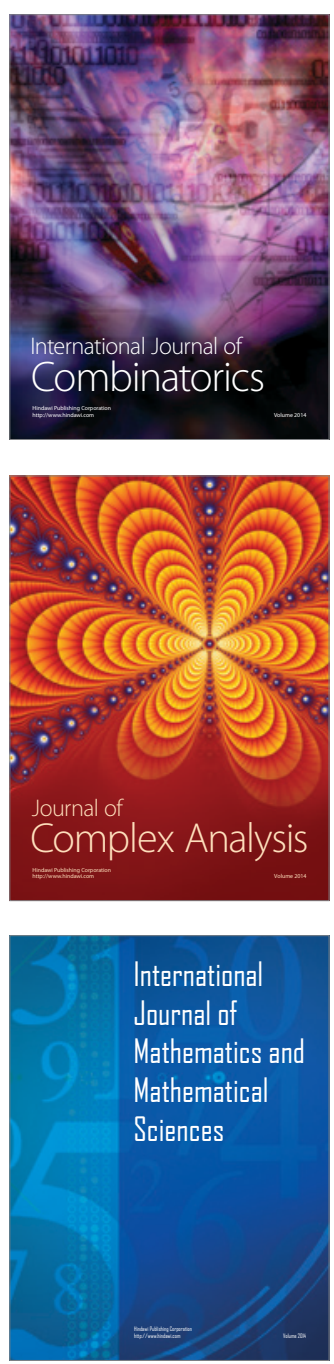
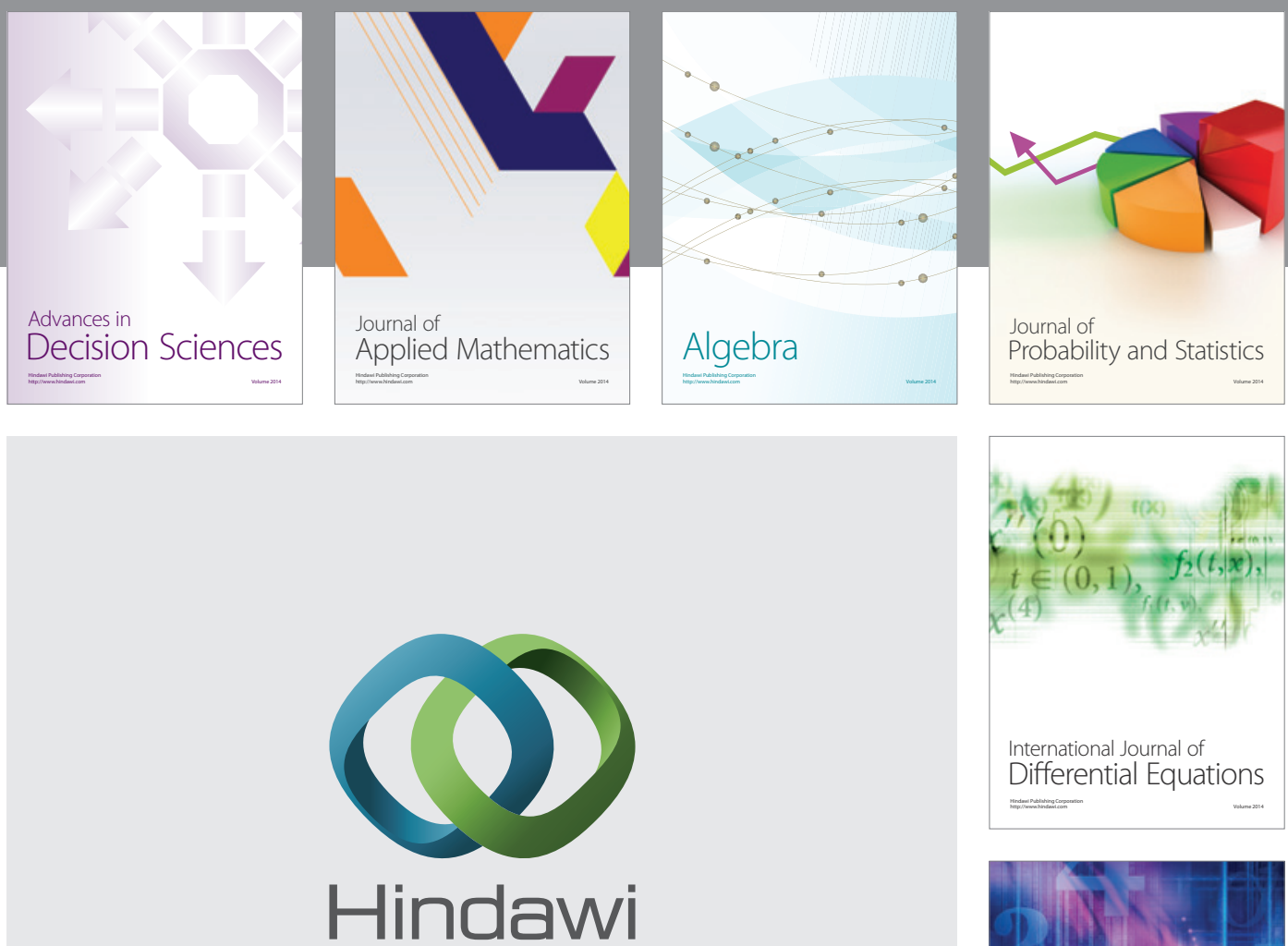

Submit your manuscripts at http://www.hindawi.com
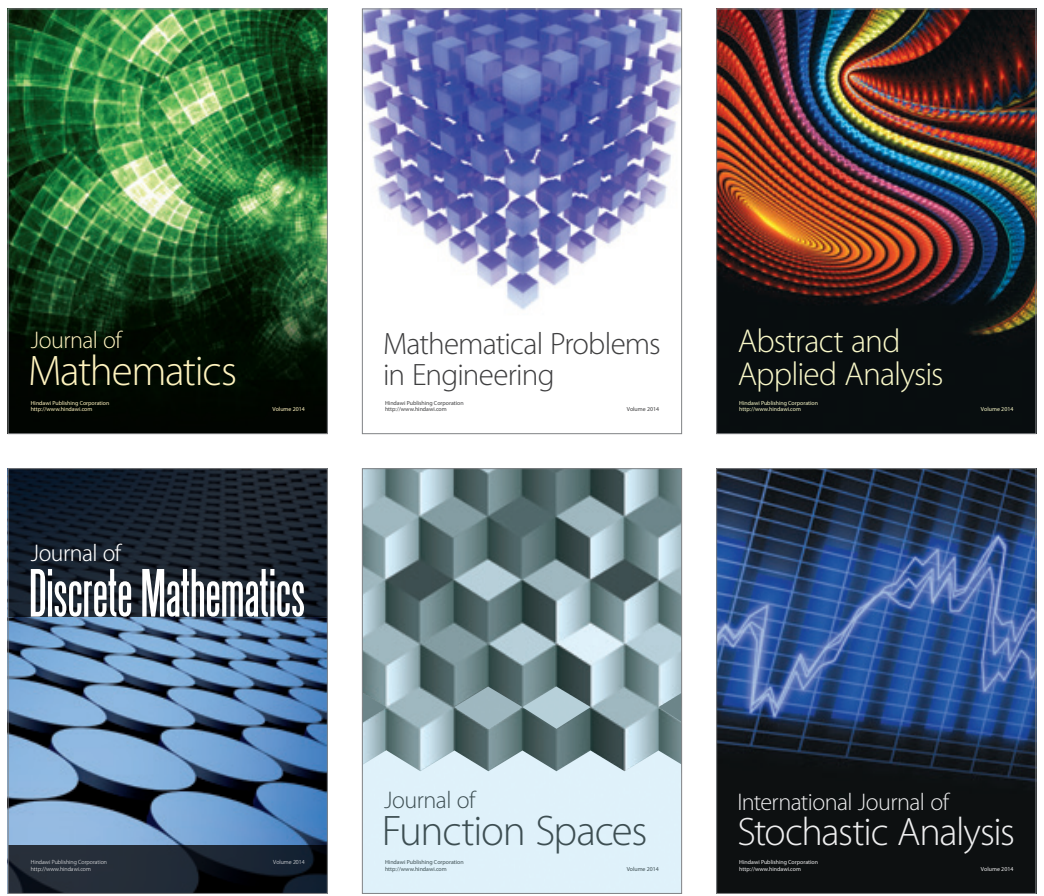

Journal of

Function Spaces

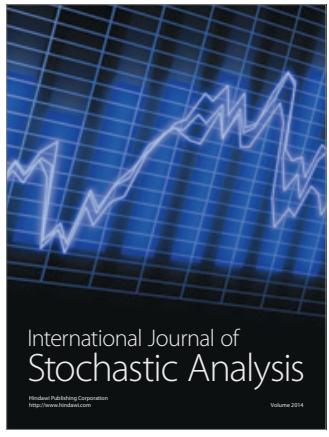

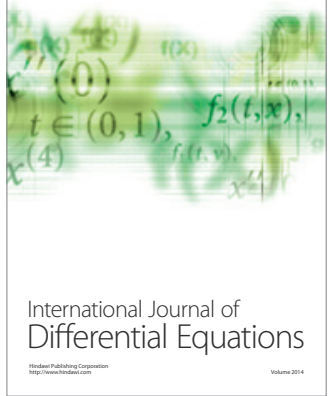
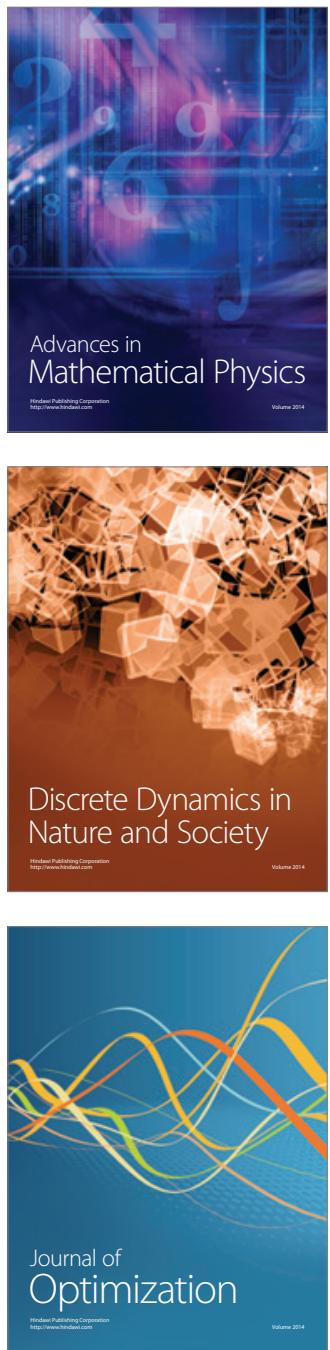